been cloned in Stanford and is being used for the analysis of this entire region of the genome by S. Beckendorf (University of California, Berkeley) and for detailed studies of the gene itself by M. Muskavitch (Stanford). A surprising result is that the transcript of SGS-4 differs in size in different strains of Drosophila. This results, it would appear, from the fact that within the coding sequence there is a 21 base pair sequence whose repetition frequency can vary between different SGS-4 alleles. Although nothing is known of the chemistry of the protein this must reflect a sequence of seven amino acids repeated a varying number of times.

The mutation characterised by Korge, that fails to make SGS-4 protein when homozygous turns out to be a small deletion, but of a sequence over 200 base pairs away from the 5 ' end of the messenger RNA. The importance of this region is indicated by the fact that two different expressed alleles of SGS-4 differ by a single base pair substitution in this region and by a factor of two in the abudance of their mRNAs.

The egg shell protein genes, under study by A. Spradling (Carnegie Institution, Washington) and in F. C. Kafatos' laboratory (Harvard) also surprised us for they are amplified in the tissue in which they are expressed. Spradling has found that the amount of DNA amplified extends at least $30 \mathrm{~kb}$ on each side of the genes themselves, but the degree of amplification falls off, from a peak of some 60 fold, in both directions away from the coding sequences. This, and the fact that the amplified DNA has an identical restriction pattern to non-amplified DNA, may indicate that the process is chromosomal, rather than extra-chromosomal as in oocyte ribosomal genes in many organisms. Yet the Harvard laboratory (G. Thiroes and R. Griffin-Shea) have found that amplification of another pair of egg shell protein genes is accompanied by their premature transcription; but that these early transcripts are not translated and, indeed, decay as development gets under way. Only at the appropriate developmental stage several hours later does transcription resume to produce mRNAs that are subsequently translated.

What is the meaning of this? It implies some relationship between amplification and early transcription and also indicates the existence of a translational control at the early developmental stage.

Like all good meetings, at the end there were far more questions than answers. As is right and proper we were treated to many very preliminary data whose place in the jigsaw puzzle is yet undiscovered. The great success of the Kolymbari meeting was in bringing together molecular and classical Drosophila biologists to discuss their data from their different perspectives and we can only hope that some of today's mysteries will be clearer to us when we next meet.

\title{
Comets and the origin of life
}

\section{from N.J. McNaughton and C.T. Pillinger}

COMETS are the most spectacular bodies in our solar system and the anticipated return of Halley's Comet in 1985-6 has already proved a catalyst for increased cometary research. As the comet nucleus contains, perhaps with the exception of phosphorus, the necessary ingredients for life on this planet, the pundits of eathbound chemical evolution and cometary science found common ground at a recent colloquium* entitled 'Comets and the origin of life'.

Although almost all of our observations of comets have been obtained from or near the Earth, the accumulated data yields a basic understanding of the structure, chemistry and physics of the comet nucleus and tails. Perhaps the most significant point to emerge from new observations is the apparent constancy of comet's chemical composition.

A'Hearn (University of Maryland) observed that the rate of production of certain chemical species, particularly $\mathrm{CN}$, $\mathrm{C}_{2}, \mathrm{C}_{3}$ and $\mathrm{OH}$, from the comet nucleus near the sun is uniform irrespective of the type of comet and its gas to dust ratio. The spectroscopic continuums of comets of different ages are also strikingly similar (Donn, NASA-Goddard). It is not yet clear whether this uniformity is due to the nature of the chemical reactions in the coma or if it implies that comets are derived from a constant composition reservoir. How the latter interpretation fits with a recently announced theory for the origin of comets (Biermann Royal Society Meeting on Planetary Exploration, Munich, Nov, 4-5, 1980 ) is not clear. Biermann's new hypothesis predicts cometisimals as the

N.J. McNaughton and C.T. Pillinger are in the Department of Earth Sciences, University of Cambridge.

$0028-0836 / 80 / 500540-01 \$ 01.00$ possible end preduct of a fragment of protosolar nebula collapsing in a relatively low magnetic flux. According to the same theory, planetary systems would be produced in a large magnetic flux and there seems to be no way for both planets and comets to be produced together.

The current interest in comets has promoted a shift in emphasis among the prebiotic synthesis investigators - low temperatures and photon or proton irradiation appear to be the order of the day. Reproducing cometary compositions and environments in the laboratory, the Maryland group and Greenberg (Leiden University, Netherlands) have synthesised 'life-like' organic molecules. Long chain hydrocarbons, nitrogenous compounds and carboxylic acids have been identified spectroscopically among the products of reactions carried out at temperatures as low as $15^{\circ} \mathrm{K}$. Given that similar molecules could only be produced on the Earth under a strongly reducing protoatmosphere (which the Levine group from Virginia calculate would be short-lived due to the high reactivity and abundance of $\mathrm{OH}$ ), the introduction of such molecules via comets into a less drastic protoatmosphere offers a real, and in some cases preferred, mechanism for the beginning of life on Earth. Speculations on this theme range from the controversial - Wickramnsinghe (University of Cardiff) envisaging comets as the carriers of already living organisms - to efforts to calculate possible mass inputs (Lizcano-Aranjo, Houston).

Definitive evidence on the relationship between comets and the origin of life must

"The Fifth College Park Colloquium on Chemical Evolution University of Maryland, Oct 29-31, 1980. await the eventual laboratory analysis of cometary material. The identification of microscopic stratospheric and deep-sea particles of extraterrestrial origin by Brownlee (University of Washington) offers, however, the first real samples of probable cometary debris. The minute size and rarity of unaltered Brownlee particles preclude the use of most conventional analytical procedures, and substantial refinements to existing or even new techniques (such as the $\mathrm{CD}_{4}$ carbon isotopic method pioneered at the University of Cambridge), are required before even the Brownlee particles can be fully exploited.

The alternative to analysing cometary material found in the terrestrial environment is in situ measurements from spacecraft. The planned NASA/ESA international mission to rendezvous with Halley's Comet which would have allowed analyses as sophisticated as those needed for origin of life studies will now not take place. ESA's fast flyby of Halley (the Giotto mission) will undoubtedly produce information on the composition of the gas and solid phases to fuel further laboratory based studies but is unlikely to provide definite answers to the question of whether comets were involved in the origin of life on Earth.

\section{CORRIGENDUM}

Edward Herbert would like to point out that he was not the sole author of Biosynthesis and processing of cellular and viral polyproteins (News and Views, 13 November, 288, 115, 1980 ) and would like to acknowledge the coauthorship of Gunther Kreil (Salzburg) and the help of Carolyn Carter and Eckard Wimmer (Stoneybrook, New York). 